\title{
Treatment for acute anterior cruciate ligament tear: five year outcome of randomised trial
}

\author{
(c) (1) (8) OPEN ACCESS
}

\author{
Richard B Frobell associate professor ${ }^{1}$, Harald P Roos associate professor ${ }^{1}$, Ewa M Roos professor ${ }^{2}$, \\ Frank W Roemer associate professor ${ }^{34}$, Jonas Ranstam professor $^{1}$, L Stefan Lohmander professor ${ }^{125}$ \\ 'Department of Orthopaedics, Clinical Sciences Lund, Lund University, Sweden; ${ }^{2}$ Institute of Sports Science and Clinical Biomechanics, University \\ of Southern Denmark, Odense, Denmark; ${ }^{3}$ Department of Radiology, University of Erlangen, Erlangen, Germany; ${ }^{4}$ Quantitative Imaging Center \\ (QIC), Department of Radiology, Boston University School of Medicine, Boston, MA, USA; ${ }^{5}$ Department of Orthopaedics and Traumatology, University \\ of Southern Denmark, Odense, Denmark
}

\begin{abstract}
Objective To compare, in young active adults with an acute anterior cruciate ligament $(\mathrm{ACL})$ tear, the mid-term (five year) patient reported and radiographic outcomes between those treated with rehabilitation plus early ACL reconstruction and those treated with rehabilitation and optional delayed $\mathrm{ACL}$ reconstruction.

Design Extended follow-up of prospective randomised controlled trial. Setting Orthopaedic departments at two hospitals in Sweden.

Participants 121 young, active adults (mean age 26 years) with acute $A C L$ injury to a previously uninjured knee. One patient was lost to five year follow-up.

Intervention All patients received similar structured rehabilitation. In addition to rehabilitation, 62 patients were assigned to early $\mathrm{ACL}$ reconstruction and 59 were assigned to the option of having a delayed $A C L$ reconstruction if needed.
\end{abstract}

Main outcome measure The main outcome was the change from baseline to five years in the mean value of four of the five subscales of the knee injury and osteoarthritis outcome score $\left(\mathrm{KOOS}_{4}\right)$. Other outcomes included the absolute $\mathrm{KOOS}_{4}$ score, all five KOOS subscale scores, SF-36, Tegner activity scale, meniscal surgery, and radiographic osteoarthritis at five years.

Results Thirty (51\%) patients assigned to optional delayed ACL reconstruction had delayed $A C L$ reconstruction (seven between two and five years). The mean change in $\mathrm{KOOS}_{4}$ score from baseline to five years was 42.9 points for those assigned to rehabilitation plus early $\mathrm{ACL}$ reconstruction and 44.9 for those assigned to rehabilitation plus optional delayed reconstruction (between group difference 2.0 points, $95 \%$ confidence interval -8.5 to $4.5 ; P=0.54$ after adjustment for baseline score). At five years, no significant between group differences were seen in $\mathrm{KOOS}_{4}(\mathrm{P}=0.45)$, any of the KOOS subscales $(\mathrm{P} \geq 0.12)$, SF-36 $(P \geq 0.34)$, Tegner activity scale $(P=0.74)$, or incident radiographic osteoarthritis of the index knee $(P=0.17)$. No between group differences were seen in the number of knees having meniscus surgery $(P=0.48)$ or in a time to event analysis of the proportion of meniscuses operated on $(P=0.77)$. The results were similar when analysed by treatment actually received.

Conclusion In this first high quality randomised controlled trial with minimal loss to follow-up, a strategy of rehabilitation plus early ACL reconstruction did not provide better results at five years than a strategy of initial rehabilitation with the option of having a later ACL reconstruction. Results did not differ between knees surgically reconstructed early or late and those treated with rehabilitation alone. These results should encourage clinicians and young active adult patients to consider rehabilitation as a primary treatment option after an acute ACL tear.

Trial registration Current Controlled Trials ISRCTN84752559.

\section{Introduction}

Acute anterior cruciate ligament rupture is a common and serious knee injury in the young active population. ${ }^{1}$ The relative importance of surgical reconstruction and rehabilitation for the short and long term outcome is debated. ${ }^{23}$ Acute anterior cruciate ligament injury may lead to unsatisfactory knee function, decreased activity, and poor knee related quality of life, and many patients with a torn anterior cruciate ligament develop osteoarthritis of the knee irrespective of treatment. ${ }^{14-6}$ In young active adults with an acute anterior cruciate ligament tear in a previously uninjured knee, we found no difference in the patient reported outcomes after two years in a randomised controlled trial comparing two treatment strategies: structured rehabilitation plus early anterior cruciate ligament reconstruction or the same structured rehabilitation with the option of having a later reconstruction if needed. ${ }^{7}$ We here report the five year patient reported and radiographic outcomes and surgical 
treatments in an extended follow up of this randomised controlled trial (the KANON trial). Our report represents the first mid-term study comparing the treatment strategy of early surgical reconstruction of a torn anterior cruciate ligament with that of structured rehabilitation and optional later reconstruction.

\section{Methods \\ Participants}

We enrolled active adults, 18 to 35 years of age, with an anterior cruciate ligament tear not more than four weeks old to a previously uninjured knee at the departments of orthopaedics at Skåne University Hospital and Helsingborg Hospital, Sweden (table $1 \Downarrow$ ). Major exclusion criteria were professional athletes (10 on the Tegner activity score; $0-10$, lowest to highest activity level), less than moderately active people ( $<5$ on the same scale), a total collateral ligament rupture, a full thickness cartilage lesion visualised on magnetic resonance imaging, and extensive meniscal fixation. Details of the recruitment process, full inclusion and exclusion criteria, and the randomisation process have been published. ${ }^{78}$ All patients provided fully informed written consent to participate in the study.

\section{Treatment}

All patients followed a similar rehabilitation protocol consistent with the consensus in the literature. ${ }^{7}$ In addition to rehabilitation, patients randomised to early anterior cruciate ligament reconstruction had a reconstruction performed within 10 weeks of injury by one of four senior knee surgeons, each of whom did more than 40 anterior cruciate ligament reconstructions annually. Those randomised to rehabilitation with the option of delayed reconstruction had anterior cruciate ligament reconstruction by the same surgeons when presenting with symptomatic knee instability as determined by the study protocol (that is, instability induced by anterior cruciate ligament insufficiency in combination with a positive pivot shift test). ${ }^{7}$ All reconstructions were single bundle, done by using either a patella tendon or hamstrings tendon procedure depending on the surgeon's preference. ${ }^{7}$ In randomised trials, these two methods have resulted in similar outcomes. ${ }^{1011}$

In both groups, meniscal tears were treated with partial resection or fixation when indicated by magnetic resonance imaging findings, clinical signs, or both. ${ }^{7}$ Patients treated with early or delayed anterior cruciate ligament reconstruction had additional meniscal surgery if unstable meniscal tears were identified at the reconstruction. We report the number of index knees with meniscus surgery over the five year period and the proportion of meniscuses operated on (assuming two intact meniscuses in every index knee before injury) by using a "time to event" analysis. In addition, we report the proportion of meniscuses operated on and the total number of meniscus surgeries (including repeat surgery on either meniscus) at five years as exploratory outcomes in the supplementary appendix.

\section{Patient reported outcomes}

Patient reported outcomes included the knee injury and osteoarthritis outcome score (KOOS), ${ }^{12}{ }^{13}$ the Medical Outcomes Study 36-item short-form health survey (SF-36) ${ }^{14}$ and the Tegner activity scale. ${ }^{15}$ Consistent with our previous publication, ${ }^{7}$ the main outcome of this report was the change from baseline to five years in the mean value of four of the five KOOS subscales $\left(\mathrm{KOOS}_{4}\right)$. Other outcomes include the crude $\mathrm{KOOS}_{4}$ score, all five separate KOOS subscales, the scores on the SF-36 physical and mental components (all 0-100 scales, worst to best), and the Tegner activity scale score (1-10, lowest to highest) at five years. In addition, we report return to pre-injury activity level as patients who reported similar or higher Tegner activity scale scores at five years compared with their pre-injury scores.

\section{Mechanical stability}

We report knee stability measures as the number of knees without signs of mechanical instability on the manual Lachman and pivot shift tests.

\section{Knee radiography and grading of images}

We obtained frontal plane posteroanterior radiographs plus sagittal plane radiographs of the tibiofemoral compartment and patella axial radiographs of the patellofemoral compartment in weight bearing at baseline and at five years. We used a standardised method to obtain patellofemoral radiographs, ${ }^{16}$ and tibiofemoral radiographs were obtained by using a slightly modified method of the Lyon-Schuss view in which patients were standing with equal weight bearing on each leg. ${ }^{17}$ The big toe, hips, and knees were pressed towards the table, and the $\mathrm{x}$ ray beam was parallel to the medial foot and tangential to the medial tibial condyle. We used fluoroscopic guidance or snapshots to align posterior and anterior aspects of the medial tibial rim.

For the injured (index) knee, one experienced musculoskeletal radiologist (FWR), who was unaware of the treatment allocation and clinical data, graded baseline and five year radiographs for osteophytes (tibiofemoral and patellofemoral compartments) and joint space narrowing (tibiofemoral compartment only) according to the Osteoarthritis Research International atlas. ${ }^{18} 19$ The intra-reader reliability (weighted $\kappa$ ) for atlas based scoring was reported as 0.67 (95\% confidence interval 0.53 to 0.82 ). ${ }^{20}$

We report presence of radiographic osteoarthritis separately for the tibiofemoral and patellofemoral compartments. In agreement with previous reports, we considered radiographic osteoarthritis to be present if any of the following criteria were achieved in either of the medial or lateral tibiofemoral compartments or in the patellofemoral compartment: joint space narrowing grade 2 or above (tibiofemoral compartment only); sum of the two marginal osteophyte grades from the same compartment 2 or above; grade 1 joint space narrowing in combination with grade 1 osteophytes in the same compartment (tibiofemoral compartment only). ${ }^{21-25}$ For the tibiofemoral compartment, this cut-off approximates to grade 2 radiographic osteoarthritis based on the Kellgren and Lawrence scale. ${ }^{26}$

\section{Statistical analysis}

A detailed description of the definition of the sample size in the randomised controlled trial has been previously reported. ${ }^{7} \mathrm{We}$ considered inclusion of 120 patients sufficient to provide $80 \%$ power to detect the requisite 10 point difference in the primary outcome. ${ }^{7}$ We present the KOOS and SF-36 scores as means and $95 \%$ confidence intervals; we present the Tegner activity scale as medians and interquartile ranges. We analysed between group comparisons by using analysis of covariance (KOOS and SF-36 scores), the Mann-Whitney U test, and the $\chi^{2}$ test for all dichotomous variables. We compared the total number of meniscus surgeries (including repeat surgery on same meniscus) and the proportion of meniscuses operated on in the two groups by using the binomial test for two Poisson distributed variables. We also used a time to event analysis to analyse the proportion of meniscuses operated on. We used IBM SPSS Statistics 19.0 for all analyses. 


\section{Results \\ Characteristics of patients and treatment of torn anterior cruciate ligament}

Despite several contacts by mail and telephone, one patient assigned to early anterior cruciate ligament reconstruction did not attend the five year clinical visit. Consequently, we included 120/121 participants in this five year follow-up report, 61 of whom were assigned to early anterior cruciate ligament reconstruction and 59 to initial rehabilitation with the option of having a later reconstruction if needed (table $1 \Downarrow$ ). ${ }^{7}$ Two patients assigned to early reconstruction did not have reconstruction or had less than 10 rehabilitation visits; we excluded them from the as treated analysis but included them in the full analysis set. Thirty (51\%) patients assigned to initial rehabilitation with the option of having a later reconstruction chose to have (and had) a delayed anterior cruciate ligament reconstruction over the five year period (seven in the period between two and five years; median 867 (range 743-1695) days after injury) (fig $1 \Downarrow$ ). Fifteen anterior cruciate ligaments were reconstructed using patella tendon procedures and 15 using hamstrings tendon procedures. Over the five year period, 61 patients had an early anterior cruciate ligament reconstruction, 30 had a delayed reconstruction, and 29 were treated with rehabilitation alone. Of the 91 primary reconstructions performed, 40 used patella tendon procedures and 51 used hamstrings tendon procedures.

\section{Patient reported outcomes}

In the full analysis set, the mean change in $\mathrm{KOOS}_{4}$ score from baseline to five years was 42.9 points for patients assigned to rehabilitation plus early anterior cruciate ligament reconstruction and 44.9 points for those assigned to rehabilitation plus optional delayed reconstruction (between group difference 2.0 points, $95 \%$ confidence interval -8.5 to $4.5 ; \mathrm{P}=0.54$ after adjustment for the baseline score). We found no statistically significant differences in $\mathrm{KOOS}_{4}$, any of the five individual subscales of KOOS, SF-36, or Tegner activity scale between the two treatment strategies at five years or in the change between two and five years (table $2 \Downarrow$ ). We found similar results in the as treated analysis (table $3 \Downarrow$ ).

\section{Mechanical stability}

Knee stability at rest at five years was statistically significantly better in knees assigned to early anterior cruciate ligament reconstruction compared with those assigned to initial rehabilitation with the option of having a later reconstruction if needed, as indicated by normal Lachman $(\mathrm{P}<0.001)$ and pivot shift $(\mathrm{P}<0.001)$ tests $($ table $2 \Downarrow)$. We found similar differences in the as treated analysis (table $3 \Downarrow$ ).

\section{Radiographic osteoarthritis}

Weight bearing radiographs were available for 113 index knees (58 assigned to early anterior cruciate ligament reconstruction and 55 assigned to initial rehabilitation with the option of having a later reconstruction if needed). Two baseline radiographs were lost in a computer crash, three patients did not attend for radiographs, and three were pregnant at the five year visit. None of the index knees had radiographic osteoarthritis at baseline. At five years, we found no statistically significant differences between the treatment groups in the full analysis set or in the as treated analysis (table $4 \Downarrow$ ). The index knee of $13(12 \%)$ patients had developed tibiofemoral radiographic osteoarthritis, and $22(19 \%)$ had developed patellofemoral radiographic osteoarthritis (six knees had concomitant tibiofemoral and patellofemoral radiographic osteoarthritis). In the full analysis set, 19 of the patients with radiographic osteoarthritis of the index knee (10 patellofemoral, 5 tibiofemoral, and 4 patellofemoral and tibiofemoral combined) were assigned to early anterior cruciate ligament reconstruction and $10(6,2$, and 2 , respectively) were assigned to initial rehabilitation with the option of having a later reconstruction if needed.

Of the 87 knees with radiographs and treated with reconstruction, done early or as delayed procedures, 10 (11\%) had developed tibiofemoral radiographic osteoarthritis and 20 (23\%) had developed patellofemoral radiographic osteoarthritis, whereas the corresponding frequency of tibiofemoral and patellofemoral radiographic osteoarthritis among the 26 knees that were treated with rehabilitation alone was $3(12 \%)$ and 2 $(8 \%)$. In the as treated analysis, we found no statistically significant differences in tibiofemoral radiographic osteoarthritis between those who had anterior cruciate ligament reconstruction (early or as delayed procedures) and those who received rehabilitation alone $(\mathrm{P}=0.995$ for tibiofemoral radiographic osteoarthritis; $\mathrm{P}=0.084$ for patellofemoral radiographic osteoarthritis). We found statistically significantly more patellofemoral radiographic osteoarthritis $(\mathrm{P}=0.001)$, but not tibiofemoral radiographic osteoarthritis $(\mathrm{P}=0.269)$, in knees reconstructed using patella tendon procedures compared with hamstrings tendon procedures.

\section{Meniscus surgery during the five year follow-up period}

Sixty one (51\%) knees, 29 treated with early anterior cruciate ligament reconstruction and 32 treated with initial rehabilitation with the option of a later reconstruction, had meniscus surgery over the five year period, with no statistically significant difference between the groups $(\mathrm{P}=0.483)$. We found no statistically significant differences in a time to event analysis of the proportion of meniscuses operated on for the full analysis set $(\mathrm{P}=0.774$; fig $2 \Downarrow)$ or in the as treated comparison $(\mathrm{P}=0.210$; fig $3 \Downarrow)$. When we accounted for repeated surgery on the same meniscus, we found a lower frequency of meniscus surgery procedures in patients treated with rehabilitation plus early anterior cruciate ligament reconstruction compared with those treated with initial rehabilitation with the option of having a later reconstruction (supplementary appendix).

\section{Anterior cruciate ligament graft ruptures and revision surgery}

Four patients had a graft rupture over the five years, of whom two had a revision anterior cruciate ligament reconstruction (both assigned to early reconstruction) and two declined revision surgery (one assigned to early anterior cruciate ligament reconstruction and one assigned to delayed reconstruction if needed).

\section{Discussion}

After five years in this randomised controlled trial, we found no statistically significant differences in pain, symptoms, function in activities of daily living, function in sports and recreation, knee related quality of life, general physical or mental health status, current physical activity level, return to pre-injury activity level, radiographic osteoarthritis, or meniscus surgery between patients assigned to rehabilitation plus early anterior cruciate ligament reconstruction and those assigned to initial rehabilitation with the option of having a later reconstruction if needed. Over the five year study period, $30(51 \%)$ of those assigned to initial rehabilitation had delayed anterior cruciate 
ligament reconstruction (seven patients between two and five years). This frequency is higher than that reported at 15 years' follow-up in a previous randomised controlled trial comparing surgical and non-surgical treatment of anterior cruciate ligament injury, ${ }^{27}$ but differences in patient selection and criteria for need of surgery confound comparisons.

\section{Comparison with other studies}

Patient reported outcomes for comparison are scarce, but our KOOS results are comparable to the five year data from the Swedish anterior cruciate ligament registry (1465 patients, all treated with reconstruction) (personal communication, $\mathrm{M}$ Forssblad, 20 April 2012), and slightly worse than the six year result from a US cohort study of anterior cruciate ligament reconstruction. ${ }^{28}$ Direct comparisons are confounded by differences in study design and loss to follow-up, but the similarities in outcomes support the generalisability of our study.

Anterior cruciate ligament reconstruction is commonly recommended for people wishing to return to pre-injury sporting activities. ${ }^{1}$ However, a recent meta-analysis of 5770 patients with anterior cruciate ligament reconstruction suggested that after a mean of 41 months' follow-up, only $63 \%$ had returned to their pre-injury activity level and $44 \%$ to competitive sports. ${ }^{29}$ At five years, our study confirms a modest return to pre-injury activity level and importantly shows that no statistically significant differences existed in return to pre-injury activity between patients treated with early or delayed anterior cruciate ligament reconstruction or with rehabilitation alone.

This randomised controlled trial was unblinded at two years, but our original study protocol pre-defined an analysis of incident radiographic osteoarthritis after five years on the basis of the well known risk of development of this problem. ${ }^{25627}$ We identified $12 \%$ of the knees as presenting with tibiofemoral radiographic osteoarthritis and $20 \%$ with patellofemoral radiographic osteoarthritis at five years after an acute anterior cruciate ligament injury, with no statistically significant differences between the groups in the full analysis set or in the as treated comparison. Inconsistencies in the acquisition and assessment of radiographs, in definitions of radiographic osteoarthritis, and in descriptions of patient cohorts make comparisons between reports difficult. ${ }^{5}$ However, we here used similar radiographic techniques and definitions of radiographic osteoarthritis as in our previous anterior cruciate ligament injury cohort studies, which found $51 \%$ radiographic osteoarthritis in men and $41 \%$ in women at $12-14$ years' follow-up, with no statistically significant difference between those reconstructed or not. ${ }^{30}{ }^{31}$ A 10 year follow-up of a case-control study using radiographic osteoarthritis classifications similar to ours found $48 \%$ radiographic osteoarthritis in knees treated with anterior cruciate ligament reconstruction and $28 \%$ in knees treated without reconstruction, with no statistically significant difference between the groups. ${ }^{2}$ Furthermore, no treatment related differences in frequency of radiographic osteoarthritis were found in a 15 year follow-up of a randomised controlled trial comparing surgical and non-surgical treatment of anterior cruciate ligament injury. ${ }^{27}$ As reported, ${ }^{32}{ }^{33}$ we found patellofemoral radiographic osteoarthritis to be more frequent in knees with reconstruction (performed early or as delayed procedures) with patella tendon grafts compared with hamstring tendon grafts. Postoperative shortening of the patellar tendon, which may lead to altered biomechanical loading of the patellofemoral compartment, correlates with the severity of patellofemoral radiographic osteoarthritis. ${ }^{33}$ Bone remodelling at the patellar harvest site may further contribute to osteophyte formation. The high frequency of radiographic osteoarthritis of the knee already at five years after anterior cruciate ligament injury at an age of about 30 is striking, and the results of this study indicate that surgical reconstruction of the torn anterior cruciate ligament does not protect the injured knee from developing structural changes consistent with osteoarthritis.

In this study, three of the $61(5 \%)$ knees treated with early anterior cruciate ligament reconstruction, and one (3\%) of the 30 knees treated with delayed reconstruction had a new severe injury resulting in a graft rupture over the five year period. These results are consistent with the $4.3 \%$ graft rupture rate reported from a five year follow-up of 1415 patients treated with anterior cruciate ligament reconstruction. ${ }^{34}$ The frequency of secondary meniscus injury or meniscus surgery after anterior cruciate ligament injury is not known. Retrospective observational studies have suggested that longer times between anterior cruciate ligament injury and reconstruction result in an increase in secondary meniscus injury. ${ }^{35-38}$ However, these reports are confounded by indication, compromising interpretation of their findings. ${ }^{39}$ In our prospective randomised controlled trial with minimal loss to follow-up, we found no statistically significant difference in the number of knees having meniscus surgery over the first five years after anterior cruciate ligament injury between treatments, either for the full analysis set or between the as treated groups. We also did a time to event analysis of the proportion of meniscuses treated with meniscus surgery and did not detect any treatment related differences. Meniscus lesions and resection are associated with an increased risk of radiographic osteoarthritis. ${ }^{514041}$ Some observational studies suggest that a meniscus lesion in an anterior cruciate ligament deficient knee increases the risk of subsequent radiographic osteoarthritis and that an anterior cruciate ligament reconstruction decreases the risk of meniscus tears. ${ }^{6}$ However, in this randomised trial we found no difference in incident radiographic osteoarthritis at five years between knees surgically reconstructed early or late and those treated with rehabilitation alone. Continued and careful long term monitoring of this and other cohorts of patients treated with or without surgical reconstruction of the anterior cruciate ligament is needed to clarify the relation between treatment of the anterior cruciate ligament injured knee, associated injuries, and long term patient reported and structural outcomes.

\section{Conclusions}

In this five year prospective trial, we have shown that in young, active adults with an acute anterior cruciate ligament tear, a strategy of early reconstruction plus rehabilitation did not provide better results, whether measured as patient reported outcomes, radiographic osteoarthritis, or meniscus surgery, than a strategy of initial rehabilitation with the option of having a later anterior cruciate ligament reconstruction. Using the second strategy, $50 \%$ of the patients did not need a reconstruction. Results at five years did not differ between knees surgically reconstructed early or late or those treated with rehabilitation alone. Our results do not apply to professional athletes or to less than moderately active people, but they should encourage clinicians and young active adult patients to consider rehabilitation as a primary treatment option after an acute anterior cruciate ligament tear. Over the five year observation time of this trial, we did not find any evidence of one treatment being more harmful than the other. Additional randomised controlled trials and large prospective long term cohort studies including surgically and non-surgically treated patients will contribute to our understanding of this common and disabling condition. 


\section{What is already known on this topic}

The relative efficacy of surgical reconstruction and rehabilitation for the short term and long term outcomes after anterior cruciate ligament (ACL) rupture is debated

\section{What this study adds}

This is the first high quality randomised controlled trial comparing surgical and non-surgical treatment strategies in young active adults with an acute ACL injury

At five years, patients assigned to rehabilitation plus early $\mathrm{ACL}$ reconstruction did not differ significantly in patient reported or radiographic outcomes from those assigned to initial rehabilitation with the option of a later reconstruction

These results should encourage clinicians and young active adult patients to consider rehabilitation as a primary treatment option following an acute $\mathrm{ACL}$ tear

We express our sincere gratitude to all patients who participated in this trial. We also acknowledge the physiotherapists who managed the rehabilitation: Idrottskliniken \& Idrottskadecentrum in Helsingborg, Höganäs Fysio and Marianne Roos in Ängelholm, Kupolen in Klippan, Idrottsskademottagningen in Landskrona, Hanspeter Borgman in Eslöv, Idrottshälsan in Lund, Rehab \& Friskvårdshuset in Staffanstorp, Mats Gustavsson at Davidshalls rehab, and Tina Magnusson at Fysiocenter in Malmö; and the orthopaedic surgeons: Ola Olsson in Helsingborg, Lars Wahlström in Ängelholm, and Fredrik Nyquist in Malmö who together with HPR performed surgical interventions. Special thanks go to Kerstin Åkesson for study management, Björn Slaug and Ludvig Dahl for database management, Jan-Åke Nilsson for statistical analysis, and Torsten Boegård for radiological advice.

Contributors: RBF, HPR, EMR, and LSL were responsible for the study design and protocol. RBF and HPR recruited and followed up patients and collected data. RBF, JR, LSL, and EMR were responsible for the statistical analysis plan. RBF, JR, LSL, FR, and EMR analysed and interpreted data. RBF and LSL wrote the manuscript, and all authors reviewed it and approved the final version. LSL is the guarantor. Funding: The KANON study received funding from the Swedish Research Council (RBF, LSL, EMR), Medical Faculty of Lund University (LSL, EMR), Region Skåne (LSL, RBF, EMR), Thelma Zoegas Fund (RBF, HPR), Stig \& Ragna Gorthon Research Foundation (RBF), Swedish National Centre for Research in Sports (LSL, RBF), Crafoord Foundation (RBF), Tore Nilsson Research Fund (RBF), and Pfizer Global Research (LSL).

Competing interests: All authors have completed the Unified Competing Interest form at www.icmje.org/coi_disclosure.pdf (available on request from the corresponding author) and declare: no support from any organisation for the submitted work other than those listed above under Funding; LSL has received honorariums for lectures from Pfizer; no other relationships or activities that could appear to have influenced the submitted work.

Ethical approval: This study was approved by the IRB at Lund University, Sweden (LU 535-2001).

Data sharing: No additional data available.

Spindler KP, Wright RW. Clinical practice: anterior cruciate ligament tear. N Engl J Med 2008;359:2135-42.

2 Meuffels DE, Favejee MM, Vissers MM, Heijboer MP, Reijman M, Verhaar JA. Ten year follow-up study comparing conservative versus operative treatment of anterior cruciate ligament ruptures: a matched-pair analysis of high level athletes. Br J Sports Med 2009;43:347-51.

3 Moksnes $\mathrm{H}$, Risberg MA. Performance-based functional evaluation of non-operative and operative treatment after anterior cruciate ligament injury. Scand J Med Sci Sports 2009;19:345-55

4 Spindler KP, Warren TA, Callison JC Jr, Secic M, Fleisch SB, Wright RW. Clinical outcome at a minimum of five years after reconstruction of the anterior cruciate ligament. $J$ Bone Joint Surg Am 2005;87:1673-9.

5 Lohmander LS, Englund PM, DahI LD, Roos EM. The long-term consequence of ACL and meniscus injuries: osteoarthritis. Am J Sports Med 2007;35:1756-69.

6 Oiestad BE, Engebretsen L, Storheim K, Risberg MA. Knee osteoarthritis after anterior cruciate ligament injury: a systematic review. Am J Sports Med 2009;37:1434-43.

7 Frobell RB, Roos EM, Roos HP, Ranstam J, Lohmander LS. A randomized trial of treatment for acute anterior cruciate ligament tears. N Engl J Med 2010;363:331-42.

8 Frobell RB, Lohmander LS, Roos EM. The challenge of recruiting patients with anterior cruciate ligament injury of the knee into a randomized clinical trial comparing surgical and non-surgical treatment. Contemp Clin Trials 2007;28:295-302.
9 Risberg MA, Lewek M, Snyder-Mackler L. A systematic review of evidence for anterior cruciate ligament rehabilitation: how much and what type? Phys Ther Sport 2004;5:125-45.

10 Biau DJ, Tournoux C, Katsahian S, Schranz PJ, Nizard RS. Bone-patellar tendon-bone autografts versus hamstring autografts for reconstruction of anterior cruciate ligament: meta-analysis. BMJ 2006;332:995-1001.

11 Spindler KP, Kuhn JE, Freedman KB, Matthews CE, Dittus RS, Harrell FE Jr. Anterior cruciate ligament reconstruction autograft choice: bone-tendon-bone versus hamstring: does it really matter? A systematic review. Am J Sports Med 2004;32:1986-95.

12 Roos EM, Roos HP, Ekdahl C, Lohmander LS. Knee injury and Osteoarthritis Outcome Score (KOOS)—validation of a Swedish version. Scand J Med Sci Sports 1998:8:439-48.

13 Roos EM, Roos HP, Lohmander LS, Ekdahl C, Beynnon BD. Knee Injury and Osteoarthritis Outcome Score (KOOS) - development of a self-administered outcome measure. J Orthop Sports Phys Ther 1998;28:88-96.

14 Ware JE Jr, Sherbourne CD. The MOS 36-item short-form health survey (SF-36). I: Conceptual framework and item selection. Med Care 1992;30:473-83.

15 Tegner Y, Lysholm J. Rating systems in the evaluation of knee ligament injuries. Clin Orthop 1985:43-9.

16 Boegard TL, Rudling O, Petersson IF, Jonsson K. Joint space width of the tibiofemoral and of the patellofemoral joint in chronic knee pain with or without radiographic osteoarthritis: a 2-year follow-up. Osteoarthritis Cartilage 2003;11:370-6.

17 Brandt KD, Mazzuca SA, Conrozier T, Dacre JE, Peterfy CG, Provvedini D, et al. Which is the best radiographic protocol for a clinical trial of a structure modifying drug in patients with knee osteoarthritis? J Rheumatol 2002;29:1308-20.

18 Altman RD, Gold GE. Atlas of individual radiographic features in osteoarthritis, revised. Osteoarthritis Cartilage 2007;15(suppl A):1-56

19 Altman RD, Hochberg M, Murphy WA Jr, Wolfe F, Lequesne M. Atlas of individual radiographic features in osteoarthritis. Osteoarthritis Cartilage 1995;3(suppl A):3-70.

20 Gossec L, Jordan JM, Mazzuca SA, Lam MA, Suarez-Almazor ME, Renner JB, et al. Comparative evaluation of three semi-quantitative radiographic grading techniques for knee osteoarthritis in terms of validity and reproducibility in 1759 X-rays: report of the OARSI-OMERACT task force. Osteoarthritis Cartilage 2008;16:742-8.

21 Englund M, Lohmander LS. Risk factors for symptomatic knee osteoarthritis fifteen to twenty-two years after meniscectomy. Arthritis Rheum 2004;50:2811-9.

22 Paradowski PT, Englund M, Roos EM, Lohmander LS. Similar group mean scores, but large individual variations, in patient-relevant outcomes over 2 years in meniscectomized subjects with and without radiographic knee osteoarthritis. Health Qual Life Outcomes 2004;2:38

23 Roos EM, Bremander AB, Englund M, Lohmander LS. Change in self-reported outcomes and objective physical function over 7 years in middle-aged subjects with or at high risk of knee osteoarthritis. Ann Rheum Dis 2008:67:505-10.

24 Englund M, Paradowski PT, Lohmander LS. Association of radiographic hand osteoarthritis with radiographic knee osteoarthritis after meniscectomy. Arthritis Rheum 2004;5:469-75.

25 Englund M, Lohmander LS. Patellofemoral osteoarthritis coexistent with tibiofemoral osteoarthritis in a meniscectomy population. Ann Rheum Dis 2005;64:1721-6.

26 Kellgren JH, Lawrence JS. Radiological assessment of osteo-arthrosis. Ann Rheum Dis 1957;16:494-502.

27 Meunier A, Odensten M, Good L. Long-term results after primary repair or non-surgical treatment of anterior cruciate ligament rupture: a randomized study with a 15-year follow-up. Scand J Med Sci Sports 2007;17:230-7.

28 Spindler KP, Huston LJ, Wright RW, Kaeding CC, Marx RG, Amendola A, et al. The prognosis and predictors of sports function and activity at minimum 6 years after anterior cruciate ligament reconstruction: a population cohort study. Am J Sport Med 2011;39:348-59.

29 Ardern CL, Webster KE, Taylor NF, Feller JA. Return to sport following anterior cruciate ligament reconstruction surgery: a systematic review and meta-analysis of the state of play. Br J Sports Med 2011;45:596-606.

30 Lohmander LS, Östenberg A, Englund M, Roos H. High prevalence of knee osteoarthritis, pain, and functional limitations in female soccer players twelve years after anterior cruciate ligament injury. Arthritis Rheum 2004;50:3145-52.

31 Von Porat A, Roos EM, Roos $\mathrm{H}$. High prevalence of osteoarthritis 14 years after an anterio cruciate ligament tear in male soccer players: a study of radiographic and patient relevant outcomes. Ann Rheum Dis 2004;63:269-73.

32 Neuman P, Kostogiannis I, Friden T, Roos H, Dahlberg LE, Englund M. Patellofemoral osteoarthritis 15 years after anterior cruciate ligament injury-a prospective cohort study. Osteoarthritis Cartilage 2009;17:284-90.

33 Jarvela T, Paakkala T, Kannus P, Jarvinen M. The incidence of patellofemoral osteoarthritis and associated findings 7 years after anterior cruciate ligament reconstruction with a bone-patellar tendon-bone autograft. Am J Sport Med 2001;29:18-24.

34 Shelbourne KD, Gray T, Haro M. Incidence of subsequent injury to either knee within 5 years after anterior cruciate ligament reconstruction with patellar tendon autograft. $A m \mathrm{~J}$ Sport Med 2009;37:246-51.

35 Church S, Keating JF. Reconstruction of the anterior cruciate ligament: timing of surgery and the incidence of meniscal tears and degenerative change. $J$ Bone Joint Surg $\mathrm{Br}$ 2005;87:1639-42.

36 Granan LP, Bahr R, Lie SA, Engebretsen L. Timing of anterior cruciate ligament reconstructive surgery and risk of cartilage lesions and meniscal tears: a cohort study 
based on the Norwegian National Knee Ligament Registry. Am J Sports Med 2009;37:955-61.

37 Tayton E, Verma R, Higgins B, Gosal H. A correlation of time with meniscal tears in anterior cruciate ligament deficiency: stratifying the risk of surgical delay. Knee Surg Sports Traumatol Arthrosc 2009;17:30-4.

38 Yoo JC, Ahn JH, Lee SH, Yoon YC. Increasing incidence of medial meniscal tears in nonoperatively treated anterior cruciate ligament insufficiency patients documented by serial magnetic resonance imaging studies. Am J Sports Med 2009;37:1478-83.

39 Van de Velde SK, Gill TJ. Treatment for acute anterior cruciate ligament tear. N Engl J Med 2010;363:1871-2, author reply 72-3.

40 Englund M, Guermazi A, Gale D, Hunter DJ, Aliabadi P, Clancy M, et al. Incidental meniscal findings on knee MRI in middle-aged and elderly persons. N Engl J Med 2008;359:1108-15.

41 Englund M, Guermazi A, Roemer FW, Aliabadi P, Yang M, Lewis CE, et al. Meniscal tear in knees without surgery and the development of radiographic osteoarthritis among middle-aged and elderly persons: the Multicenter Osteoarthritis Study. Arthritis Rheum 2009;60:831-9.

Accepted: 28 December 2012

\section{Cite this as: BMJ 2013;346:f232}

This is an open-access article distributed under the terms of the Creative Commons Attribution Non-commercial License, which permits use, distribution, and reproduction in any medium, provided the original work is properly cited, the use is non commercial and is otherwise in compliance with the license. See: http://creativecommons.org/licenses/bync/2.0/ and http://creativecommons.org/licenses/by-nc/2.0/legalcode. 


\section{Tables}

Table 1| Baseline characteristics of study patients. Values are numbers (percentages) unless stated otherwise

\begin{tabular}{|c|c|c|c|c|c|c|c|}
\hline \multirow[b]{2}{*}{ Characteristics } & \multicolumn{3}{|c|}{ Full analysis set } & \multicolumn{4}{|c|}{ As treated groups } \\
\hline & $\begin{array}{c}\text { Early } A C L \\
\text { reconstruction } \\
(n=61)\end{array}$ & $\begin{array}{l}\text { Delayed optional } \\
\text { ACL } \\
\text { reconstruction } \\
(n=59)\end{array}$ & $P$ value & $\begin{array}{c}\text { Early } A C L \\
\text { reconstruction } \\
(n=59)\end{array}$ & $\begin{array}{c}\text { Delayed ACL } \\
\text { reconstruction } \\
\quad(n=30)\end{array}$ & $\begin{array}{l}\text { Rehabilitation } \\
\text { alone } \\
(n=29)\end{array}$ & $P$ value \\
\hline $\begin{array}{l}\text { Mean }(\mathrm{SD}) \text { age } \\
\text { (years) }\end{array}$ & $26.4(5.1)$ & $25.8(4.7)$ & 0.47 & $26.6(5.1)$ & $25.2(4.5)$ & $26.4(4.9)$ & 0.47 \\
\hline Female sex & $12(20)$ & $20(34)$ & 0.08 & $12(20)$ & $11(37)$ & $9(31)$ & 0.23 \\
\hline $\begin{array}{l}\text { Mean (SD) body } \\
\text { mass index }\end{array}$ & $24.5(3.1)$ & $23.8(2.6)$ & 0.22 & $24.5(3.2)$ & $23.3(2.0)$ & $24.3(3.1)$ & 0.25 \\
\hline $\begin{array}{l}\text { Increased } \\
\text { anteroposterior laxity }\end{array}$ & $60(98)^{\star}$ & $58(98) \dagger$ & 0.98 & $58(98)^{*}$ & $29(97) \dagger$ & $29(100)$ & 0.61 \\
\hline $\begin{array}{l}\text { Median (interquartile } \\
\text { range) Tegner } \\
\text { activity scale }\end{array}$ & $9(7-9)$ & $9(7-9)$ & 0.82 & $9(7-9)$ & $8.5(7-9)$ & $9(7-9)$ & 0.91 \\
\hline
\end{tabular}

$\mathrm{ACL}=$ anterior cruciate ligament.

A comprehensive description of baseline characteristics for all included patients has been published. ${ }^{7}$

*In one knee, anteroposterior laxity could not be assessed owing to pain; magnetic resonance imaging (MRI) and arthroscopy confirmed total ACL rupture. †In one knee, anteroposterior laxity was found to be normal at baseline, but MRI and arthroscopy confirmed total ACL rupture. 
Table 2| Patient reported outcomes and mechanical knee stability measures at five years and change between two and five years for full analysis set $(n=120)$

\begin{tabular}{|c|c|c|c|c|}
\hline Outcome & $\begin{array}{l}\text { Early ACL reconstruction } \\
\qquad(\mathrm{n}=61)\end{array}$ & $\begin{array}{l}\text { Delayed optional ACL } \\
\text { reconstruction } \\
(n=59)\end{array}$ & Mean difference $(95 \% \mathrm{Cl})$ & $P$ value \\
\hline $\begin{array}{l}\text { Mean (95\% Cl) follow-up after } \\
\text { randomisation (months) }\end{array}$ & 60 (59 to 61$)$ & 59 (57 to 60$)$ & $1.2(-0.6$ to 3.0$)$ & 0.06 \\
\hline \multicolumn{5}{|l|}{ Patient reported outcomes at 5 years } \\
\hline \multicolumn{5}{|l|}{ Mean $(95 \% \mathrm{Cl}) \mathrm{KOOS}^{*}$ : } \\
\hline $\mathrm{KOOS}_{4} \dagger$ & 80 (76 to 84$)$ & 82 (77 to 86 ) & $-1.5(-7.4$ to 4.4$)$ & 0.45 \\
\hline Pain & 91 (88 to 94 ) & 91 (88 to 94$)$ & $-0.3(-4.6$ to 4.0$)$ & 0.73 \\
\hline Symptoms & 83 (78 to 87 ) & 87 (83 to 91$)$ & $-4.4(-10.2$ to 1.4$)$ & 0.12 \\
\hline Activities of daily living & 95 (93 to 98$)$ & 97 (95 to 99$)$ & $-1.5(-4.3$ to 1.4$)$ & 0.38 \\
\hline Sport and recreation & 76 (70 to 82$)$ & 79 (73 to 86$)$ & $-3.3(-11.7$ to 5.2$)$ & 0.23 \\
\hline Knee related QOL & 71 (66 to 76$)$ & 69 (63 to 75$)$ & $1.8(-6.2$ to 9.8$)$ & 0.89 \\
\hline \multicolumn{5}{|l|}{ Mean $(95 \% \mathrm{Cl})$ SF-36ł: } \\
\hline Physical component & 85 (81 to 89$)$ & $84(80$ to 89$)$ & $0.9(-4.9$ to 6.7$)$ & 0.78 \\
\hline Mental component & 87 (84 to 91$)$ & 85 (84 to 91$)$ & $2.8(-2.7$ to 8.3$)$ & 0.34 \\
\hline $\begin{array}{l}\text { Median (interquartile range) Tegner } \\
\text { activity scale§ }\end{array}$ & $4(2.5-7)$ & $4(2-7)$ & $0.1(-0.8-1.1)$ & 0.74 \\
\hline $\begin{array}{l}\text { No (\%) active at pre-injury Tegner } \\
\text { activity scale level } ₫\end{array}$ & $14(23)$ & $12(20)$ & $2.6(-12.4-17.6) \ddagger$ & 0.73 \\
\hline \multicolumn{5}{|l|}{ Mechanical knee stability at 5 years } \\
\hline No (\%) normal Lachman test ${ }^{\star *}$ & $45 / 58(76)$ & $19 / 58(33)$ & $43.5(27.0$ to 60.1$) \dagger \dagger$ & $<0.001$ \\
\hline No (\%) normal pivot shift testł‡ & $45 / 58(76)$ & $23 / 58(40)$ & $36.6(19.7$ to 53.5$) \dagger \dagger$ & $<0.001$ \\
\hline \multicolumn{5}{|c|}{ Change in patient reported outcomes (5 minus 2 years) } \\
\hline \multicolumn{5}{|l|}{ Mean $(95 \% \mathrm{Cl}) \mathrm{KOOS}$ : } \\
\hline $\mathrm{KOOS}_{4}$ & $4(1$ to 8$)$ & $5(1$ to 10$)$ & $-1.3(-6.7$ to 4.2$)$ & 0.38 \\
\hline Pain & $4(1$ to 7$)$ & $4(0$ to 7$)$ & $0.3(-3.9$ to 4.4$)$ & 0.86 \\
\hline Symptoms & $4(0$ to 8$)$ & $4(0$ to 8$)$ & $0.0(-5.5$ to 5.6$)$ & 0.87 \\
\hline Activities of daily living & $2(0$ to 4$)$ & $2(0$ to 4$)$ & $-0.2(-3.0$ to 2.6$)$ & 0.76 \\
\hline Sport and recreation & $5(1$ to 10$)$ & $8(2$ to 14$)$ & $-3.5(-11.6$ to 4.7$)$ & 0.27 \\
\hline Knee related QOL & $4(0$ to 8$)$ & $6(0$ to 12$)$ & $-2.0(-9.2$ to 5.1$)$ & 0.35 \\
\hline \multicolumn{5}{|l|}{ Mean $(95 \% \mathrm{Cl}) \mathrm{SF}-36$ : } \\
\hline Physical component & $4(-1$ to 8$)(n=60)$ & $6(3$ to 10$)$ & $-1.7(-6.9$ to 3.5$)$ & 0.12 \\
\hline Mental component & $-1(-5$ to 3$)$ & $1(-3$ to 4$)$ & $-1.7(-6.9$ to 3.5$)$ & 0.52 \\
\hline
\end{tabular}

$\mathrm{ACL}=$ anterior cruciate ligament; KOOS=knee injury and osteoarthritis outcome score; $\mathrm{QOL}=$ quality of life.

${ }^{*} \mathrm{KOOS}$ ranges from 0 to 100 , with higher scores indicating better results. ${ }^{12,13}$

†Includes four KOOS subscales: pain, symptoms, function in sports and recreation, and knee related quality of life; scores range from 0 to 100 , with higher scores indicating better results. ${ }^{\top}$

$\ddagger$ Scores range from 0 to 100 , with higher scores indicating better results. ${ }^{14}$

§Assesses activity level with specific emphasis on knee; scores range from 1 (least strenuous activity) to 10 (high knee demanding activity on professional sports level). ${ }^{15}$

IPatients with score at five years that was same as or higher than pre-injury score.

**Assesses anteroposterior laxity of knee at rest in semiflexed position; results range from 0 (normal laxity) to 3 (severely increased laxity); data include knees with normal laxity.

††95\% confidence interval for percentage.

$\ddagger \ddagger$ Assesses rotational stability of knee at rest; results range from 0 (normal stability) to 3 (severely increased instability); data include knees with normal stability. 
Table 3| Patient reported outcomes and mechanical knee stability measures at five years and change between two and five years for as treated groups $(n=118)$

\begin{tabular}{|c|c|c|c|c|c|c|c|c|c|}
\hline \multirow[b]{3}{*}{ Outcome } & \multicolumn{3}{|c|}{ As treated groups } & \multicolumn{6}{|c|}{ Mean between group differences } \\
\hline & \multirow[b]{2}{*}{$\begin{array}{c}\text { Early } A C L \\
\text { reconstruction } \\
(n=59)\end{array}$} & \multirow[b]{2}{*}{$\begin{array}{c}\text { Delayed ACL } \\
\text { reconstruction } \\
\quad(n=30)\end{array}$} & \multirow[b]{2}{*}{$\begin{array}{l}\text { Rehabilitation } \\
\text { alone } \\
(n=29)\end{array}$} & \multirow[b]{2}{*}{$\begin{array}{c}\text { Early } v \\
\text { delayed ACL } \\
\text { reconstruction }\end{array}$} & \multirow[b]{2}{*}{$P$ value } & \multirow{2}{*}{$\begin{array}{c}\text { Early ACL } \\
\text { reconstruction } \\
\mathbf{v} \\
\text { rehabilitation } \\
\text { alone }\end{array}$} & \multirow{2}{*}{\multicolumn{2}{|c|}{$\begin{array}{l}\text { Delayed ACL } \\
\text { reconstruction } \\
\mathbf{v} \\
\text { rehabilitation } \\
\text { alone }\end{array}$}} & \multirow[b]{2}{*}{$P$ value } \\
\hline & & & & & & & & & \\
\hline $\begin{array}{l}\text { Mean }(95 \% \mathrm{Cl}) \\
\text { follow-up after } \\
\text { randomisation } \\
\text { (months) }\end{array}$ & $60(59$ to 61$)$ & 59 (57 to 60$)$ & 58 (55 to 61$)$ & $1(-1$ to 2$)$ & 0.883 & $2(-1,4)$ & 0.145 & $1(-3$ to 4$)$ & 0.611 \\
\hline \multicolumn{10}{|c|}{ Patient reported outcomes at 5 years } \\
\hline \multicolumn{10}{|l|}{$\begin{array}{l}\text { Mean }(95 \% \mathrm{Cl}) \\
\text { KOOS*: }^{*}\end{array}$} \\
\hline $\mathrm{KOOS}_{4} \dagger$ & $80(75$ to 84$)$ & 81 (75 to 88$)$ & 82 (76 to 88$)$ & $-1(-9$ to 6$)$ & 0.698 & $-3(-10$ to 5$)$ & 0.492 & $-1(-10$ to 8$)$ & 0.804 \\
\hline Pain & 91 (88 to 94) & 91 (86 to 96$)$ & 92 (87 to 96$)$ & $0(-5$ to 5$)$ & 0.968 & $-1(-6$ to 4$)$ & 0.697 & $-1(-7$ to 5$)$ & 0.771 \\
\hline Symptoms & 83 (78 to 87$)$ & 85 (79 to 90$)$ & 89 (83 to 95) & $-3(-10$ to 5$)$ & 0.466 & $-7(-15$ to 0$)$ & 0.054 & $-5(-12$ to 3$)$ & 0.221 \\
\hline $\begin{array}{l}\text { Activities of } \\
\text { daily living }\end{array}$ & 95 (93 to 97$)$ & 97 (95 to 99$)$ & 96 (93 to 100$)$ & $-2(-6$ to 1$)$ & 0.246 & $-1(-5$ to 3$)$ & 0.537 & $1(-3$ to 4$)$ & 0.649 \\
\hline $\begin{array}{l}\text { Sport and } \\
\text { recreation }\end{array}$ & 76 (70 to 81$)$ & 78 (68 to 87$)$ & 81 (73 to 90$)$ & $-2(-13$ to 9$)$ & 0.712 & $-6(-16$ to 4$)$ & 0.255 & $-4(-16$ to 9$)$ & 0.536 \\
\hline $\begin{array}{l}\text { Knee related } \\
\text { QOL }\end{array}$ & 71 (65 to 76 ) & 72 (63 to 80$)$ & 66 (58 to 75$)$ & -1 (-11 to 9$)$ & 0.822 & $4(-6$ to 14$)$ & 0.401 & $5(-7$ to 17$)$ & 0.388 \\
\hline \multicolumn{10}{|l|}{$\begin{array}{l}\text { Mean }(95 \% \mathrm{Cl}) \\
\text { SF-36‡: }\end{array}$} \\
\hline $\begin{array}{l}\text { Physical } \\
\text { component }\end{array}$ & 85 (79 to 92 ) & 83 (78 to 89 ) & 85 (79 to 92 ) & $2(-5$ to 9$)$ & 0.616 & $0(-8$ to 7$)$ & 0.967 & $-2(-10$ to 7$)$ & 0.652 \\
\hline $\begin{array}{l}\text { Mental } \\
\text { component }\end{array}$ & 87 (83 to 91 ) & 86 (81 to 91$)$ & 83 (76 to 90$)$ & $1(-5$ to 8$)$ & 0.677 & $4(-3$ to 11$)$ & 0.294 & $3(-6$ to 11$)$ & 0.543 \\
\hline $\begin{array}{l}\text { Median } \\
\text { (interquartile } \\
\text { range) Tegner } \\
\text { activity scale§ }\end{array}$ & 4 (2 to 7 ) & 4 (2 to 7 ) & 4 (2 to 6.5$)$ & $0(-1$ to 1$)$ & 0.734 & $0(-1$ to 1$)$ & 0.959 & $0(-2$ to 1$)$ & 0.949 \\
\hline $\begin{array}{l}\text { No (\%) active at } \\
\text { pre-injury Tegner } \\
\text { activity scale } \\
\text { levelף }\end{array}$ & $13(22)$ & $6(20)$ & $6(21)$ & $2(-17 \text { to } 18)^{\star \star}$ & 0.828 & $1(-18 \text { to } 18)^{\star *}$ & 0.915 & $-1(-22 \text { to } 20)^{\star \star}$ & 0.924 \\
\hline \multicolumn{10}{|c|}{ Mechanical knee stability at 5 years } \\
\hline $\begin{array}{l}\text { No (\%) normal } \\
\text { Lachman test†† }\end{array}$ & $45(76)$ & $18(60)$ & $1 / 28(4)$ & $16(-3 \text { to } 36)^{\star *}$ & 0.121 & $72(52 \text { to } 82)^{\star \star}$ & $<0.001$ & $56(32 \text { to } 71)^{* *}$ & $<0.001$ \\
\hline $\begin{array}{l}\text { No (\%) normal } \\
\text { pivot shift testłł }\end{array}$ & $45(76)$ & $18(60)$ & $5 / 28(18)$ & $16(-3 \text { to } 36)^{\star \star}$ & 0.121 & $58(38 \text { to } 72)^{* *}$ & $<0.001$ & $42(17 \text { to } 60)^{\star *}$ & 0.002 \\
\hline \multicolumn{10}{|c|}{ Change in patient reported outcomes ( 5 minus 2 years) } \\
\hline \multicolumn{10}{|l|}{$\begin{array}{l}\text { Mean }(95 \% \mathrm{Cl}) \\
\text { KOOS: }\end{array}$} \\
\hline $\mathrm{KOOS}_{4}$ & 4 (1 to 8$)$ & $8(0$ to 15$)$ & $3(-1$ to 8$)$ & $-3(-11$ to 4$)$ & 0.337 & $1(-5$ to 7$)$ & 0.754 & $4(-4$ to 13$)$ & 0.308 \\
\hline Pain & 4 (1 to 7 ) & $5(0$ to 11$)$ & $2(-1$ to 5$)$ & $-1(-7$ to -4$)$ & 0.648 & $2(-2$ to 7$)$ & 0.363 & $3(-3$ to 10$)$ & 0.302 \\
\hline Symptoms & $4(0$ to 8$)$ & $4(-2$ to 11$)$ & $3(-2$ to 9$)$ & $-1(-8$ to 6$)$ & 0.856 & $0(-6$ to 7$)$ & 0.900 & $1(-7$ to 9$)$ & 0.789 \\
\hline $\begin{array}{l}\text { Activities of } \\
\text { daily living }\end{array}$ & $2(-1$ to 4$)$ & $3(0$ to 6$)$ & $2(0$ to 4$)$ & $-1(-5$ to 3$)$ & 0.725 & $0(-3$ to 4$)$ & 0.869 & $1(-2$ to 4$)$ & 0.571 \\
\hline $\begin{array}{l}\text { Sport and } \\
\text { recreation }\end{array}$ & $5(-1$ to 11$)$ & 11 (1 to 22 ) & $5(-1$ to 12$)$ & $-6(-17$ to 5$)$ & 0.254 & $-1(-10$ to 9$)$ & 0.912 & $6(-7$ to 18$)$ & 0.360 \\
\hline $\begin{array}{l}\text { Knee related } \\
\text { QOL }\end{array}$ & 4 (0 to 8$)$ & $10(0$ to 20$)$ & $2(-5$ to 9$)$ & $-6(-17$ to 5$)$ & 0.283 & $2(-6$ to 9$)$ & 0.643 & $8(-4$ to 20$)$ & 0.205 \\
\hline \multicolumn{10}{|l|}{$\begin{array}{l}\text { Mean }(95 \% \mathrm{CI}) \\
\text { SF-36: }\end{array}$} \\
\hline $\begin{array}{l}\text { Physical } \\
\text { component }\end{array}$ & $4(-1$ to 8$)$ & 9 (4 to 14$)$ & $4(-2$ to 9$)$ & -5 (-13 to 2$)$ & 0.157 & 0 (-8 to 8$)$ & 0.992 & $5(-2$ to 13$)$ & 0.153 \\
\hline
\end{tabular}


Table 3 (continued)

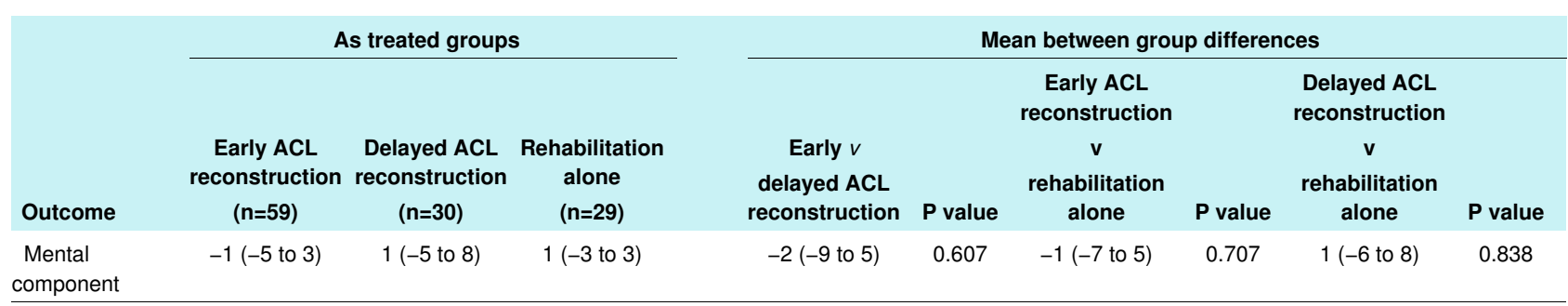

$\mathrm{ACL}=$ anterior cruciate ligament; KOOS=knee injury and osteoarthritis outcome score; $\mathrm{QOL}=$ quality of life.

*KOOS ranges from 0 to 100 , with higher scores indicating better results. ${ }^{12,13}$

†Includes four KOOS subscales: pain, symptoms, function in sports and recreation, and knee related quality of life; scores range from 0 to 100 , with higher scores indicating better results. ${ }^{7}$

$\ddagger$ Scores range from 0 to 100 , with higher scores indicating better results. ${ }^{14}$

§Assesses activity level with specific emphasis on knee; scores range from 1 (least strenuous activity) to 10 (high knee demanding activity on professional sports level). ${ }^{15}$

IPatients with score at five years that was same as or higher than pre-injury score.

${ }^{* *} 95 \%$ confidence interval for percentage.

††Assesses anteroposterior laxity of knee at rest in semiflexed position; results range from 0 (normal laxity) to 3 (severely increased laxity); data include knees with normal laxity.

$\ddagger \ddagger$ Assesses rotational stability of knee at rest; results range from 0 (normal stability) to 3 (severely increased instability); data include knees with normal stability. 
Table 4| Radiographic osteoarthritis of index knee at five years as graded by Osteoarthritis Research International atlas..$^{18,19}$ Values are numbers (percentages) unless stated otherwise

\begin{tabular}{|c|c|c|c|c|c|c|c|}
\hline \multirow[b]{2}{*}{ Five year follow-up } & \multicolumn{3}{|c|}{ Full analysis set } & \multicolumn{4}{|c|}{ As treated groups } \\
\hline & $\begin{array}{c}\text { Early } A C L \\
\text { reconstruction } \\
(n=58)\end{array}$ & $\begin{array}{l}\text { Delayed optional } \\
\text { ACL } \\
\text { reconstruction } \\
\quad(n=55)\end{array}$ & $P$ value & $\begin{array}{l}\text { Early } A C L \\
\text { reconstruction } \\
(n=58)\end{array}$ & $\begin{array}{c}\text { Delayed ACL } \\
\text { reconstruction } \\
\quad(n=29)\end{array}$ & $\begin{array}{c}\text { Rehabilitation } \\
\text { alone } \\
(n=26)\end{array}$ & $P$ value \\
\hline \multicolumn{8}{|c|}{ Tibiofemoral compartment ${ }^{*}$} \\
\hline $\begin{array}{l}\text { Radiographic } \\
\text { osteoarthritis } †\end{array}$ & $9(16)$ & $4(7)$ & 0.17 & $9(16)$ & $1(3)$ & $3(12)$ & 0.25 \\
\hline Medial compartment\$: & 6 & 3 & & 6 & 1 & 2 & \\
\hline JSN grade $\geq 2$ & - & 一 & & & & & \\
\hline Osteophytes grade $\geq 2$ & 6 & 2 & & 6 & 1 & 1 & \\
\hline $\begin{array}{c}\mathrm{JSN} \geq 1 \text { and } \\
\text { osteophytes } \geq 1\end{array}$ & - & 1 & & & & 1 & \\
\hline Lateral compartmentł: & 6 & 1 & & & - & 1 & \\
\hline JSN grade $\geq 2$ & - & - & & & & & \\
\hline Osteophytes grade $\geq 2$ & 5 & 1 & & 5 & & 1 & \\
\hline $\begin{array}{c}\text { JSN } \geq 1 \text { and } \\
\text { osteophytes } \geq 1\end{array}$ & 2 & - & & 2 & & & \\
\hline \multicolumn{8}{|c|}{ Patellofemoral compartment ${ }^{*}$} \\
\hline $\begin{array}{l}\text { Radiographic } \\
\text { osteoarthritis } \dagger \text { : }\end{array}$ & $14(24)$ & $8(15)$ & 0.20 & $14(24)$ & $6(21)$ & $2(8)$ & 0.21 \\
\hline Osteophytes grade $\geq 2$ & 14 & 8 & & 14 & 6 & 2 & \\
\hline
\end{tabular}

$\mathrm{ACL}=$ anterior cruciate ligament; $\mathrm{JSN}=$ joint space narrowing.

Osteophyte and JSN grading was performed according to Osteoarthritis Research International atlas (0-3, best to worst). ${ }^{18,19}$

*Four knees in rehabilitation and early $\mathrm{ACL}$ reconstruction group and two knees in rehabilitation plus delayed $\mathrm{ACL}$ reconstruction group (1 delayed $\mathrm{ACL}$ reconstruction and 1 rehabilitation alone) had both femorotibial and patellofemoral radiographic osteoarthritis.

†Radiographic osteoarthritis of tibiofemoral compartment was considered to be present if JSN grade $\geq 2$, and/or sum of two marginal osteophyte grades from same compartment $\geq 2$, and/or grade $1 \mathrm{JSN}$ in combination with grade 1 osteophytes in same compartment; radiographic osteoarthritis of patellofemoral compartment was considered to be present if sum of two marginal osteophyte grades was $\geq 2$.

$\ddagger$ Three knees in rehabilitation and early ACL reconstruction group had both medial and lateral compartment radiographic osteoarthritis. 


\section{Figures}

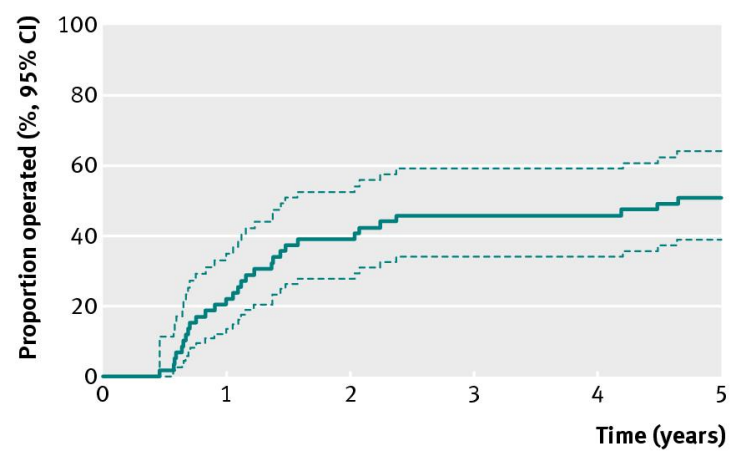

Fig 1 Cumulative proportion of patients operated on with delayed anterior cruciate ligament $(A C L)$ reconstruction for those randomised to rehabilitation plus optional delayed $A C L$ reconstruction $(n=59)$

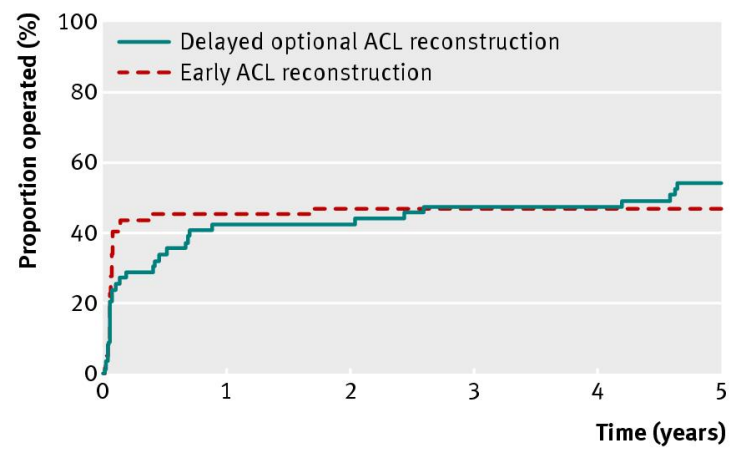

Fig 2 Proportion of meniscuses operated on in index knee (assuming two intact meniscuses in every index knee before injury) over five year follow-up period for knees treated with rehabilitation plus anterior cruciate ligament (ACL) reconstruction $(n=61)$ and knees treated with initial rehabilitation with option of later ACL reconstruction if needed ( $n=59)$ (full analysis set)

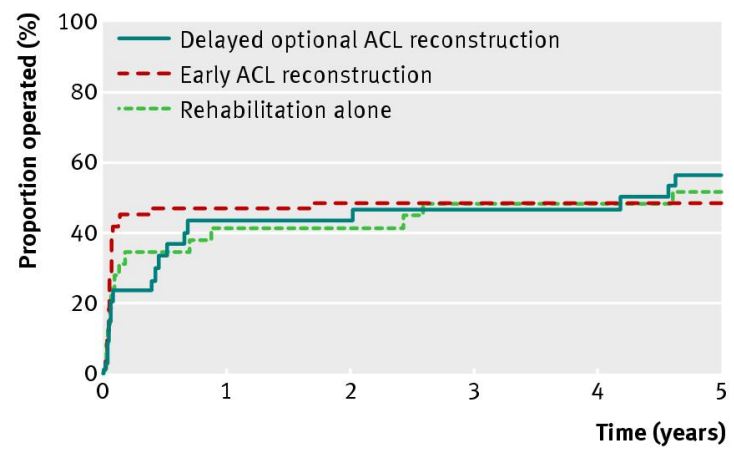

Fig 3 Proportion of meniscuses operated on in index knee (assuming two intact meniscuses in every index knee before injury) over five year follow-up period for knees treated with rehabilitation plus early anterior cruciate ligament (ACL) reconstruction $(n=60)$, knees treated with initial rehabilitation plus delayed $A C L$ reconstruction $(n=30)$, and knees treated with rehabilitation alone $(\mathrm{n}=29)$ (as treated analysis) 